\title{
Impact of Isoflurane and Sevoflurane Anesthesia on Kidney Structure and Function in Rats
}

\author{
Flavia RUXANDA ${ }^{1}$, Viorel MICLĂUŞ ${ }^{1 *}$, Vasile RUS, Adrian Florin GAL ${ }^{1}$ and \\ Liviu OANA ${ }^{1}$ \\ ${ }^{1}$ Faculty of Veterinary Medicine, University of Agricultural Sciences and Veterinary Medicine Cluj- \\ Napoca, 3-5 Mănăștur Street, 400372, Romania; \\ *Corresponding author: vmiclaus@usamvcluj.ro
}

Bulletin UASVM Veterinary Medicine 71(2) / 2014,

Print ISSN 1843-5270; Electronic ISSN 1843-5378

DOI:10.15835/buasvmcn-vm: 10388

\begin{abstract}
Various researchers studied the nephrotoxicity of inhalational anesthetics, with contradictory results. We aimed to observe the impact of the repeated isoflurane or sevoflurane anesthesia on kidney structure and function. 40 female rats were used in this study. Animals were divided in 8 groups. Two groups served as control: IM (isoflurane) and SM (sevoflurane), exposed to oxygen alone (1l/min) 3 times, for 2 hours, every other day. Three groups were anesthetized with isoflurane (I1, I2, I3) and the other three (S1, S2, S3) with sevoflurane. The anesthetics were administered three times, for two hours long each, every other day. Rats were sacrificed through cervical dislocation as follows: groups IM, SM, I1, S1 imediately after the last anesthesia, groups I2, S2 6 hours post-anesthesia and groups I3, S3 - 24 hours post-anesthesia. Kidney samples were harvested, fixed in $10 \%$ buffered formaline, embedded in paraffin, sectioned ( $5 \mu \mathrm{m}$ thickness) and stained with Goldner's trichrome metod. After isoflurane anesthesia, the kidney displays a moderate congestion in the corticomedullary area, which maintains throughout the experimental period. The only changes in animals anesthetized with sevoflurane also appear on the vascular component. The congestion maintains throughout the experimental period in the renal cortex, as well as the corticomedullary area. No other aspects that suggest functional impairment are observed, in any of the experimental groups. Inhalational anesthesia with isoflurane and sevoflurane produces a moderate congestion, without majorly impairing the kidney function.
\end{abstract}

Keywords: congestion, isoflurane, kidney, sevoflurane.

\section{INTRODUCTION}

After theinhalation anesthesia, the renal function depends on the systemic hemodynamics, renal blood flow, distribution of the renal blood flow and tubular sensitivity to fluoride (Fish, 1994). The objective quantification of the renal function impairment degree is difficult - there are many factors causing such manifestation. Many anesthesia practices and surgical procedures, like antibiotics, surgical stress, preexistent renal diseases, intraoperative blood pressure, site of intervention and anesthetics were incriminated in causing renal disfunction, but none has been evaluated in prospective studies (Ebert and Arain, 2000).

The nephrotoxicity of some anesthetic products has been assessed in some reports. Sahin etal.
(2011) observed an increase in the postoperative values of the blood urea nitrogen, creatinin and glycosuria in comparison with the preoperative ones in sevoflurane group, but without any significant differences compared with the control group.They suspected that these discrete changes of the renal function are due to the surgical stress. Eger et al. (1997) reported the fact that when the volunteers were administered sevoflurane for 2-4 hours, the markers of renal injury did not modify 2 hours after the anesthesia, but a slight albuminuria and increased $\alpha$ glutation-s-transferase (GST) were recorded. Based on these results, they claim that the threshold for renal lesions in humans is between 80 and 160 ppm-h Compound A. On the contrary, other authors have found that under the 
same experimental conditions, neither 4 , nor 8 hours of sevoflurane anesthesia have significantly affected the kidney functionality (Ebert et al., 1998a; 1998b). There were studies which evaluated the effects of prolonged anesthesia with low-flow sevoflurane on kidney function, by comparing high-flow sevoflurane anesthesia with low-flow isoflurane anesthesia. The renal function was assessed through uremia, serum creatinine, creatinine clearance and urinary excretion of glucose, albumine, protein and $\mathrm{N}$-acetil- $\beta$-Dglucosaminidase. Uremia and serum creatinine were in normal range and creatinine clearance did not decrease during the study in none of the groups. Instead, the urinary glucose excretion, albumine, protein and $\mathrm{N}$-acetil- $\beta$-D-glucosaminidase have increased after anesthesia in all groups, but no significant differences between groups were noticed at any moment. Lactate dehydrogenase and alkaline phosphatase were higher after highflow sevoflurane anesthesia. The conclusion of this experiment was that prolonged anesthesia with low-flow sevoflurane has the same effects on renal funcions as high-flow sevoflurane and lowflow isoflurane anesthesia. (Obata et al., 2000)

Karasch et al. (2001) did not observe significant differences between sevoflurane and isoflurane renal effects in surgical patients, undergoing prolonged low-flow anesthesia (for up to 17 hours). They determined the serum creatinine and blood urea nitrogen levels, the creatinine or urine protein and glucose excretion clearance. Although there were certain increases in the postoperatory values of serum creatinine, blood urea nitrogen and glycosuria levels, they were still situated within normal limits. Repeated exposure to sevoflurane and isoflurane between 30 and 180 days did not have an additional risk of increasing the urinary protein and glucose excretion in comparison with the first exposure to the same anesthetic (Nishiyama et al., 1998). Some authors have reached the conclusion that there is no evidence of nephrotoxic effects of sevoflurane in mice and rats, by studying the effects of sevoflurane anesthesia on these animals (Frink et al., 1992; Malan et al., 1993).

The aim of our study was to investigate any possible renal injures following exposure of rats to inhalation anesthetic products (isoflurane and sevoflurane).

\section{MATERIAL AND METHODS}

Animals in this experimental study were first acclimatized to the new conditions: $22-23^{\circ} \mathrm{C}$, around $60 \%$ humidity and a 12 hour light/dark cycle. The rats benefited of an ad libitum rodent pelleted diet (Cantacuzino Institute, Romania) and also water. The study was conducted in accordance with the European Union legislation on animal experimentation and with the practices of the Romanian Board of Animal Research. We also obtained the approval from the Committee of Animal Ethics at Faculty of Veterinary Medicine Cluj-Napoca, Romania.

The experimental protocol was carried out at the Discipline of Anesthesiology and Surgical Propedeutics at the University of Agricultural Sciences and Veterinary Medicine.

40 female Wistar rats ( 6 weeks old) were used in the experimental study. The rats were divided into 8 groups, each group consisting of 5 rats. Two groups served as control: IM (isoflurane control group) and SM (sevoflurane control group). The two control groups were exposed to oxygen alone (1l/min) 3 times, for 2 hours, with a 2 day interval between exposures. Three groups (I1, I2 and I3) were subdued to isoflurane anesthesia (isoflurane concentration $=1.5 \%$ ), and the other three (S1, S2 and S3) to sevoflurane anesthesia (sevoflurane concentration $=2 \%$ ). The anesthetic administration was made in 3 rounds, 2 hours each, at a 2 day interval between the administrations. The oxygen supply throughout the anesthesia was $11 \mathrm{O}_{2} / \mathrm{min}$ in both isoflurane and sevoflurane anesthesias.

The rats were sacrificed through cervical dislocation as follows: groups IM, SM, I1 and S1 immediately after the last anesthesia, groups I2 and S2 - 6 hours post-anesthesia, and groups I3 and S3 - 24 hours post-anesthesia. Kidney samples were harvested and fixed in $10 \%$ buffered formaline, embedded in paraffin, sectioned at a 5 $\mu \mathrm{m}$ thickness and stained with Goldner's trichrome method.

\section{RESULTS AND DISCUSSIONS}

Rat kidneys from control groups have a normal aspect in both renal cortex (Fig. 1) and medulla. Bowman's space, as well as the uriniferous tubules have normal sizes and do not contain a specific material that would suggest the impairment of the glomerular ultrafiltration process. 
At the completion of the isoflurane anesthesia, the kidney does not present notable changes in comparison with the control group, the only aspect that is worth taking into account is the moderate congestion of the vessels in the corticomedullary zone (Fig. 2). This congestion maintains throughout the whole experimental period, with a slight increase at 6 and 24 hours post-anesthesia.

In the case of the sevoflurane anesthetized animals, the situation is highly comparable, in the sense that the only changes appear on the vascular component. At the completion of the anesthesia, the congestion is present in the cortical zone on both small and large caliber vessels, with the highest intensity in the corticomedullary zone. The congestion maintains throughout the whole experimental period in the renal cortex (Fig. 3), as well as the passage zone between cortex and medulla (Fig. 4).

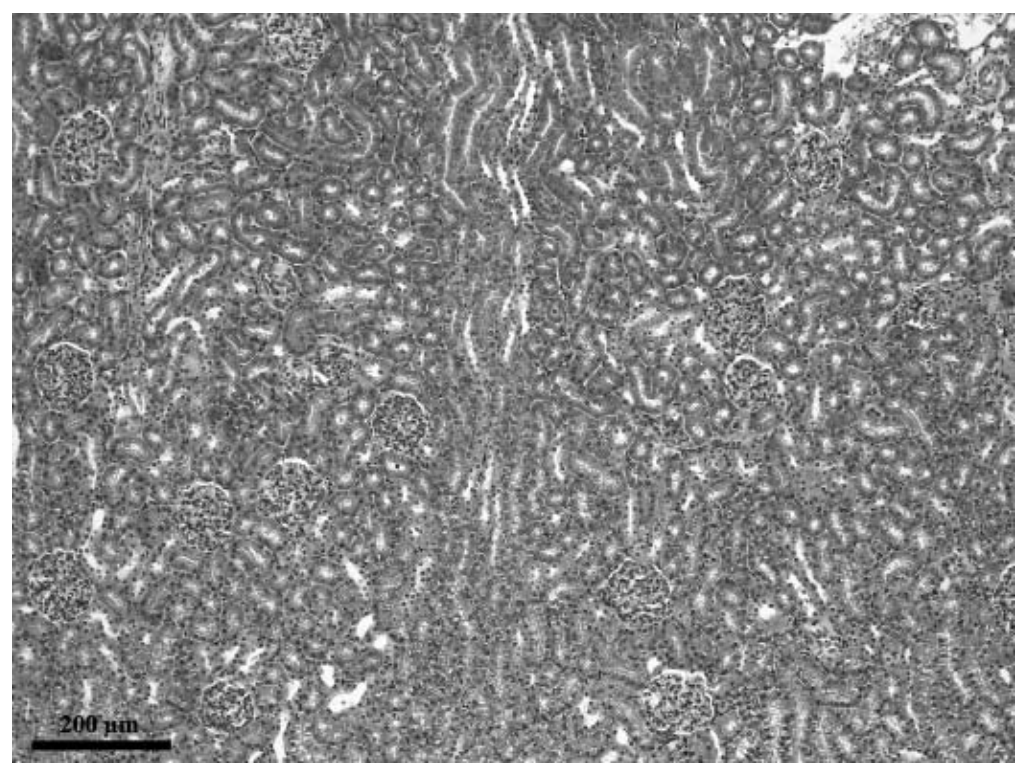

Fig. 1. Control group - Renal cortex (10X obj. Goldner's trichrome stain)

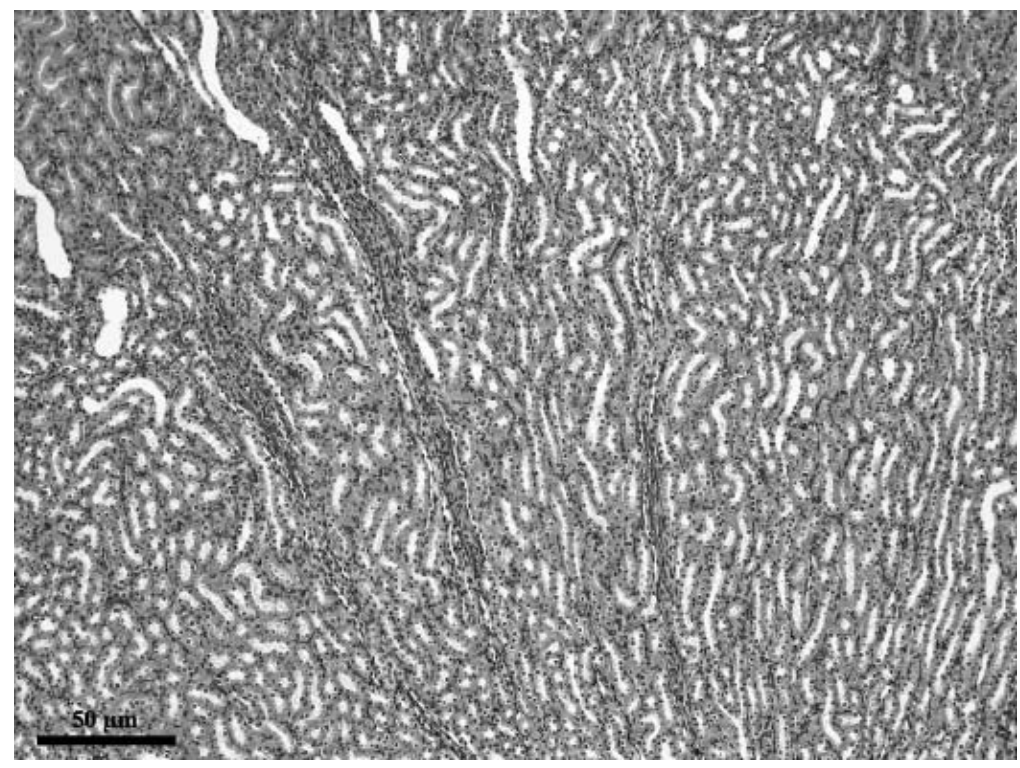

Fig. 2. Isoflurane group - Congestion in the corticomedullary zone (10X obj. Goldner's trichrome stain) 


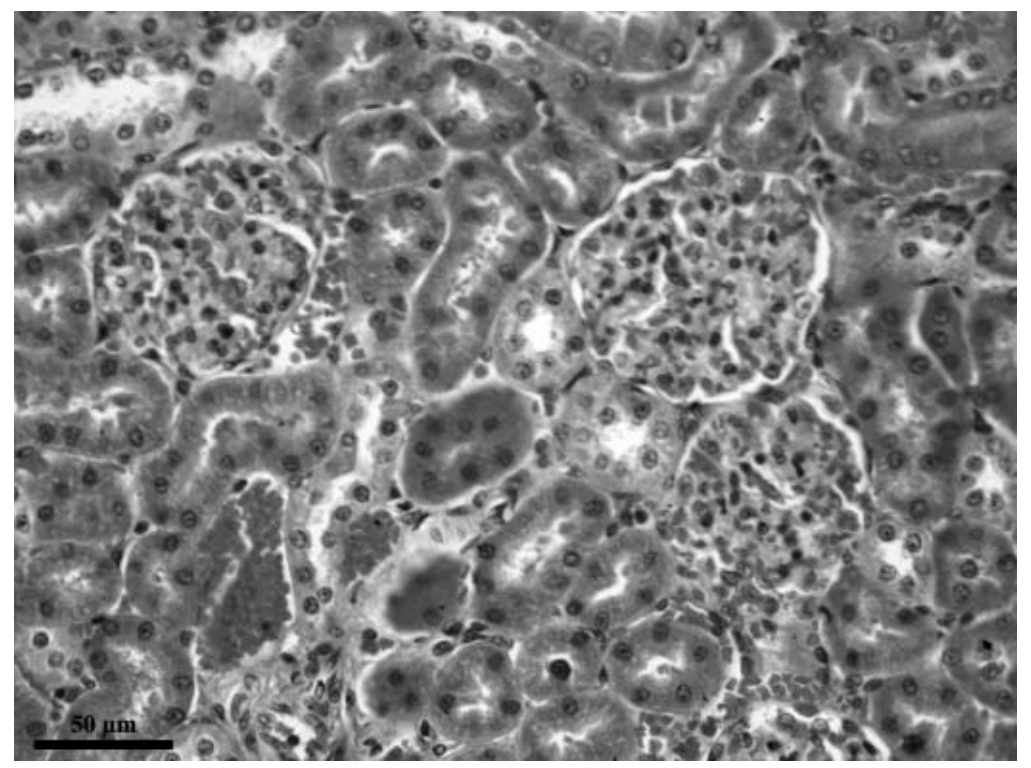

Fig. 3. Sevoflurane group - Renal cortex (40X obj. Goldner's trichrome stain)

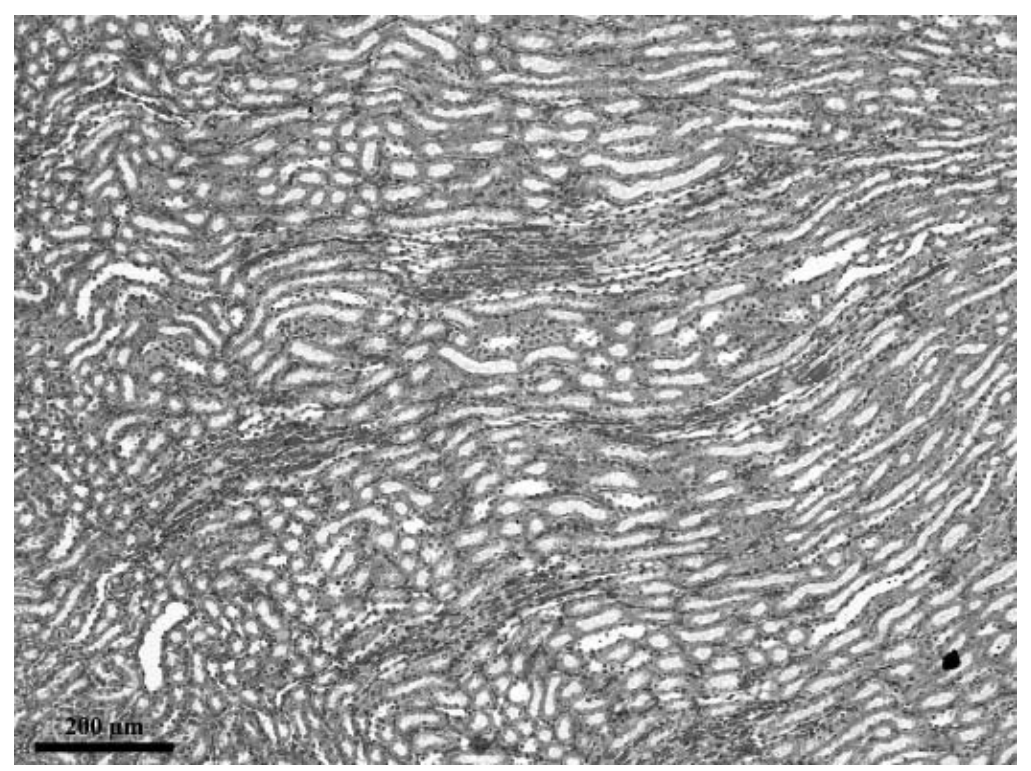

Fig. 4. Sevoflurane group - Corticomedullary zone (10X obj. Goldner's trichrome stain)

At the end of the isoflurane anesthesia, the kidney widely maintains a comparable aspect to the kidney of the rats in the control group, regarding the urine filtration components, conditioning and transport. There are no materials in neither the urinary space of Bowman's capsule, nor the lumen of uriniferous tubules to suggest the impairment of the urine filtration, reabsorbtion, concentration or transport processes. The only aspect that could result after the anesthetic action was observed on the vascular component and is represented by a relatively discrete congestion, with a more distinct expression in small vessels in the corticomedullary zone. We mention that they are not at a high intensity and are not accompanied by functional changes associated with vascular permeability (not even discrete). We observed no oedemas or other aspects that might appear in such a situation. After anesthesia, the congestion maintains in the same area (corticomedullary), slightly and 
progressively increasing in intensity at 6 and 24 hours, respectively. This aspect suggests that the anesthetic determines a discrete increase in the renal blood flow, but does not rich such an extent as to significantly impair the organ functionality, at least not in the species taken into study and at the administered dose.

The kidneys from rats anesthetized with sevoflurane also display changes only in the vascular component: a vascular ectasia present on both large and small vessels in the renal cortex, with the largest intensity on small vessels in the corticomedullary zone. The intensity of the congestion is a bit more pronounced than the one in rats anesthetized with isoflurane. The congestion is largely maintained throughout the experiment, but its intensity does not increase. Also, in the case of sevoflurane anesthesia there are no aspects to suggest kidney structure or functionality impairing.

The presenceand persistence of the congestion in the areas that contain components participating in urine filtration and conditioning, highlight the increase in renal blood flow after sevoflurane administration. This aspect could influence the glomerular filtration processes to a certain extent, namely unwinding them at a slightly higher intensity, but without exceeding the physiologic limits. This aspect could be interpreted as an effort of the organism to eliminate the anesthetic in the shortest time possible, which we consider to be benefic for the body.

Studying the potential toxic effect of repeated exposure to sevoflurane, Elena et al. (2003) observed a significant increase of the relative weight of the kidney, but without any other aspects that would betray the existence of functional impairment of the kidney, and histological studies did not reveal the existence of lesions detectable through light microscopy. Other authors have reported the increase of the relative weight of the kidneys after sevoflurane anesthesia (Basketter et al., 1995), without noticing histopathological impairments or differences in renal function, assessed through relevant pathophysiologic markers, like plasma urea and creatinine concentrations. They did not manage to find an explanation to show a potential causal link for the increased kidney weigh in treated animals. Analyzing these results, we observe that they deserve a bit of attention, because it is not enough to notice the increase in kidney weight, without trying to find a logical explanation. In our opinion, there could be only one explanation, namely, an increased blood supply to the kidney under the influence of the anesthetic, which modifies the organ weight, directly proportional to the excess blood (in comparison to the usual situation) existent at a given time. The increased blood flow does not necessarily produce structural changes and does not majorly impair the glomerular filtration processes, but eventually intensifies them to a certain extent. In our opinion, it is the only plausible explanation for the aspect reported by the forementioned authors, even if these authors did not notice it. Moreover, the investigation conducted by us, clearly highlights this aspect, because we did not observe structural changes in the renal components either, but we observed the existence of a relatively persistent congestion, present in the animals that underwent sevoflurane anesthesia. It is true that we did not weigh the kidneys, but indirectly we can state that the increased blood flow determines a certain increase of the organ weight. As long as it does not majorly impair kidney functionality, it can not be considered to be a negative aspect, especially considering that this increased blood flow is transient.

The explanation concerning the vasodilatation (at the corticomedullary junction) remains obscure for us, but it is known that the intrarenal blood flow is redistributed towards the inner cortex and medulla following ischemia or hypoperfusion. It occurs because the vasoconstrictive effects of angiotensin II. Accordingly, perfusion of outer cortical nephrons is reduced while the perfusion of inner cortical nephrons is maintained (Jubb et al., 1985). Regarding our study, it is likely that during anesthesia, an impaired oxigenation of the blood may occur that may trigger the described reaction in the kidney parenchyma.

\section{CONCLUSION}

The nephrotoxic effects of some anesthetic products have been assessed in some reports, but only a limited number of them used the histological assessment. Inhalational anaesthesia with isoflurane and sevoflurane didn't induce major histological changes in the kidney parenchyma except for a moderate vasodilatation at the corticomedullary junction. 
Acknowledgements. This paper was published under the frame of European Social Fund, Human Resources Development Operational Programme 2007-2013, projectno. POSDRU/159/1.5/S/136893

\section{REFERENCES}

1. Basketter DA, JN Bremmer, P Buckley, ME Kammuller, T Kawabata, I Kimber, S Loveless, S Magda, D Stringer, W Vohr (1995), Pathology considerations for, and subsequent risk assessment of, chemicals identified as immuno-suppressive in routine toxicology, Food and Chemical Toxicology, vol. 33, p. 239-243

2. Ebert TJ, EJ Frink, ED Kharasch (1998a), Absence of biochemical evidence for renal and hepatic dysfunction after 8 hours of 1.25 minimum alveolar concentration sevoflurane anesthesia in volunteers, Anesthesiology, vol. 88, p. $601-610$

3. Ebert TJ, LD Messana, TD Uhrich, TS Staacke (1998b), Absence of renal and hepatic toxicity after four hours of 1.25 minimum alveolar anesthetic concentration sevoflurane anesthesia in volunteers, Anesth Analg, vol. 86; p. 662-667

4. Ebert TJ, SR Arain (2000), Renal responses to lowflow desflurane, sevoflurane and propofol in patients, Anesthesiology, vol. 93, p. 1401-1406

5. Eger EI II, D Gong, DD Koblin (1997), Dose-related biochemical markers of rena injury after sevoflurane versus desflurane anesthesia in volunteers, Anesth Analg, vol. 85, p. 1154-1163

6. Elena G, N Amerio, P Ferrero, ML Bay, J Valenti, D Colluci, NR Puig (2003), Effects of repetitive sevoflurane anaesthesia on immune response, select biochemical parameters and organ histology in mice, Laboratory Animals, vol. 37, p. 193-203

7. Fish K (1994), Nephrotoxicity of volatile anaesthetic agents, In: Anesthetic Toxicity (Rice S., Fish K., eds). New York: Raven Press Ltd, 89-106

8. Frink E Jr., H Ghantous, T Malan, S Morgan, J Fernando, A Gandolfi, B Jr. Brown (1992), Plasma inorganic fluoride with sevoflurane anaesthsia: correlations with indices of hepatic and renal function, Anesthesia and Analgesia, vol. 74, p. 231-235

9. Jubb KVF, PC Kennedy, N Palmer (1985), Pathology of Domestic Animals, 3rd edition, vol. 2, Ed. Academic Press, INC., p. 240-307

10. Kharasch ED, EJ Frink, A Artru, P Michalowski, GA Rooke, W Nogami (2001), Long-duration low-flow sevoflurane and isoflurane effects on postoperative renal and hepatic function, Anesth Analg., vol. 93, p. 1511-1520

11. Malan T, G Jr. Philip, Y Kadota, H Mata, E Jr. Frink, B Jr. Brown (1993), Renal function after sevoflurane or enflurane anesthesia in Fischer 344 rats, Anesthesia and Analgesia, vol. 77, p. 817-821

12. Nishiyama T, T Yokoyama, K Hanaoka (1998), Liver and renal function after repeated sevoflurane or isoflurane anaesthesia, Can J Anaesth, vol. 45, no. 8, p. 789-793

13. Obata R, H Bito, M Ohmura, G Morwaki, Y Ikeuchi, T Katoh, S Sato (2000), The effects of prolonged low-flow sevoflurane anesthesia on renal and hepatic function, Anesth Analg, vol. 91, p. 1262-1268

14. Sahin SH, SO Cinar, I Paksoy, N Sut, S Oba (2011), Comparison between low flow sevoflurane anesthesia and total intravenous anesthesia during intermediateduration surgery: effects on renal and hepatic toxicity, Hippokratia, vol. 15, issue 1, p. 69-74 